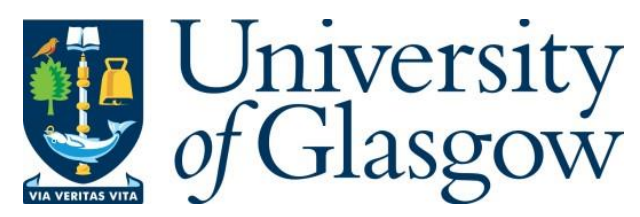

Klitis, C., Cantarella, G., Strain, M. J. and Sorel, M. (2017) High-extinction-ratio TE/TM selective Bragg grating filters on silicon-on-insulator. Optics Letters, 42(15), pp. 3040-3043.

There may be differences between this version and the published version. You are advised to consult the publisher's version if you wish to cite from it.

http://eprints.gla.ac.uk/146478/

Deposited on: 28 August 2017

Enlighten - Research publications by members of the University of Glasgow http://eprints.gla.ac.uk 


\title{
High-Extinction-ratio TE/TM selective Bragg grating filters on silicon-on-insulator
}

\author{
Charalambos Klitis $^{1,}{ }^{*}$, Giuseppe Cantarella ${ }^{2}$, Michael J. Strain ${ }^{2}$, and Marc Sorel ${ }^{1}$ \\ ${ }^{1}$ School of Engineering, University of Glasgow, Glasgo G12 8LT, UK \\ ${ }^{2}$ Institute of Photonics, University of Strathclyde, Glasgow G1 1RD, UK \\ *Corresponding author: c.klitis.1@research.gla.ac.uk
}

Compiled August 28, 2017

We report on the design and fabrication of TE and TM polarisation selective Bragg gratings filters in the form of sinusoidal perturbations on the waveguide sidewall and etched holes on the top of the waveguide respectively. Combining the two geometries on a silicon-oninsulator waveguide resulted in Bragg grating filters with high extinction ratios of approximately $60 \mathrm{~dB}$. () 2017 Optical Society of America

OCIS codes: (130.0130)Integrated optics; (130.5440) Polarizationselective devices;(230.1480) Bragg reflectors;(350.2770) Gratings.

Integrated photonics devices are extremely popular and versatile components for telecommunication systems [1], quantum photonics [2, 3],hybrid III-V on silicon lasers [4, 5] and signal processing $[6,7]$. In these applications, the high index contrast offered by the silicon-on-insulator (SOI) photonic platform is of great benefit to component miniaturisation and dense integration [8]. However, the main drawback of highly confining waveguides is a stronger scattering that still represents the main source of propagation losses. Also, scattering from non-ideal surfaces causes coupling to counter-propagating guided modes and polarisation rotation effects that severely degrades the performance of several integrated circuits [9]. One such example is the popular sidewall Bragg grating that offers a simple technological geometry to design TE polarisation filters with apodised and chirped profiles [10-12]. The strong polarisation scattering limits the maximum extinction ratio (ER) of these filters to approximately $30 \mathrm{~dB}$ [10], which prevents their exploitation in applications requiring a high ER such as quantum circuits [13-15]. Filters with higher ER values can be obtained with more complex designs such as Bragg gratings integrated with Mach-Zehnder interferometers [16] or coupled ring resonators $[15,17]$.

In this paper we present the design and evaluation of an alternative grating geometry consisting of etched holes on the top surface of the waveguide. Contrary to sidewall gratings, this solution is mostly interacting with the TM mode and therefore provides an appealing solution to selectively filter the TM polarisation only. Not only does the combination of both grating geometries greatly improves the performance of Bragg grating filters but also offers an interesting solution for on-chip polar- isation manipulation. The theoretical design of the filters will be discussed in section II, followed by device fabrication and characterisation in section III, discussion of results in section IV and conclusions in section $\mathrm{V}$.
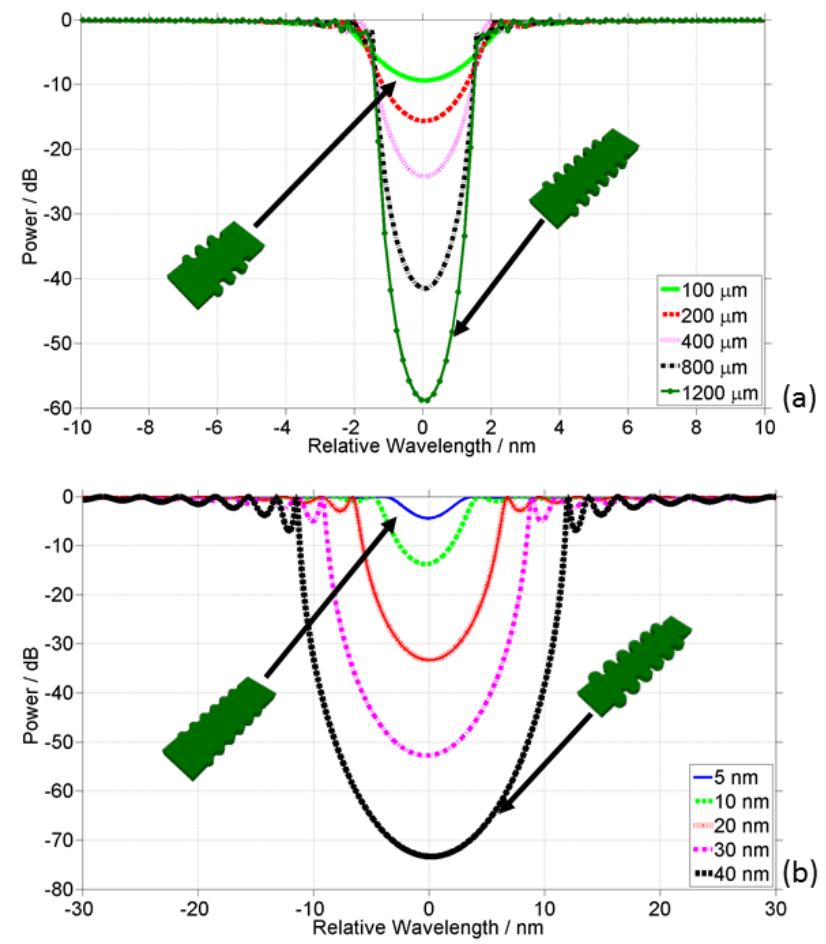

Fig. 1. Simulated optical spectra of sidewall gratings for the TE polarization on a SOI single-mode waveguide as a function of (a) grating length with a fixed grating amplitude of $5 \mathrm{~nm}$ and (b) grating amplitude with a fixed grating length $50 \mu \mathrm{m}$ [18]

In order to improve the extinction ratio of Bragg filters, one can either increase the length or the strength of the grating. Figure 1 shows the simulated spectra of a sinusoidal sidewall grating for the TE mode as a function of its length and amplitude on a single mode SOI waveguide with $220 \mathrm{~nm}$ height and 500 $\mathrm{nm}$ width. For all the simulations described in this work unless 
stated otherwise, an eigenmode expansion (EME) [19] solver under the commercial software Mode solutions from Lumerical was used [18].

Acting on both the length and amplitude of the gratings offers the ability to accurately control the ER and the stop band width of the transmission spectrum. However, sidewall gratings have almost no effect on the TM polarisation as shown by the simulated optical spectra of Figure 2. In fact, only very strong and long gratings have any measurable $\mathrm{ER}$, which is however several orders of magnitude lower than the figures obtained on the TE polarisation with identical grating parameters. Larger ERs for the TM polarisation can be theoretically achieved if longer and stronger gratings are used, however, the theoretical propagation losses for the TE mode as extracted from the EME simulations can easily exceed $10 \mathrm{~dB} / \mathrm{cm}$ if a $30 \mathrm{~dB}$ extinction ratio for the TM mode is required.
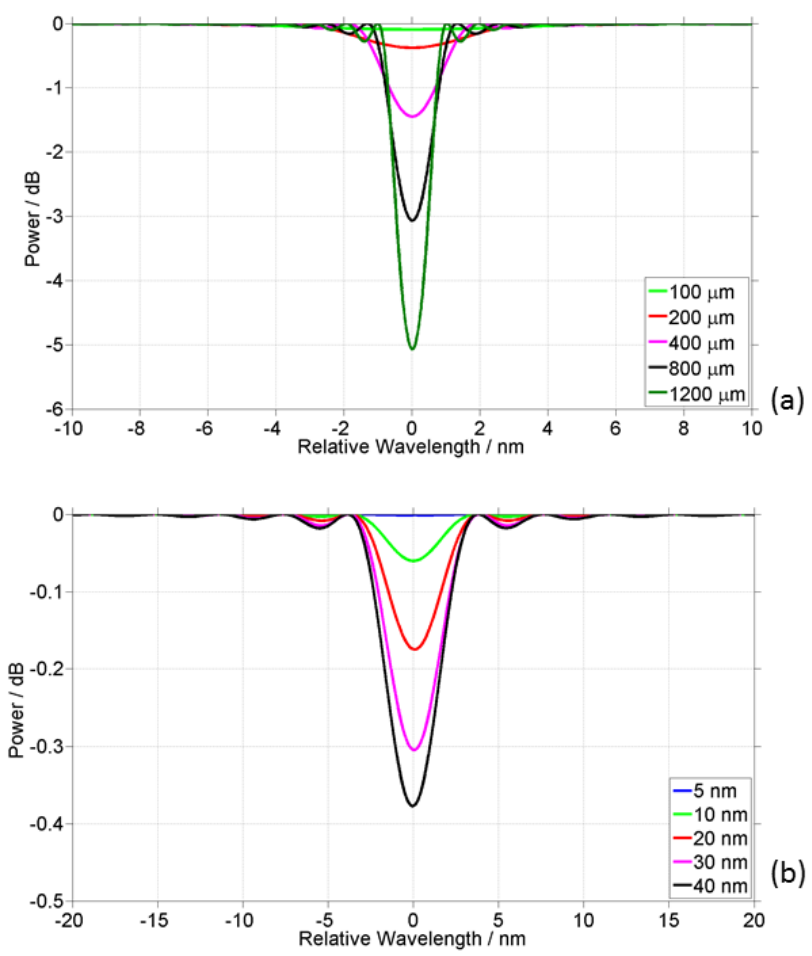

Fig. 2. The simulated optical spectra of sidewalls gratings for the TM polarisation on a SOI single-mode waveguide as a function of (a) the length with a fixed grating amplitude of 40 $\mathrm{nm}$ and (b) the grating amplitude with a fixed grating length of $200 \mu m$ [18].

The reason for such different results between the two polarisations is simply a consequence of their modal profile as depicted in the Finite Difference Eigenmode (FDE) simulations of Figure 3. While the TE polarised mode has a strong overlap with the sidewalls of the waveguide, the TM polarisation has only a very weak interaction and hence it is little influenced by the presence of sidewall gratings. On the other hand, the simulations indicate that the TM polarisation has a strong overlap with the top and the bottom of the waveguide, which suggests that a strong TM Bragg grating filter can be designed by placing the gratings on the top of the waveguide.

The shape of the top grating has been chosen in this case to be circular for easier fabrication, and hence the grating performance
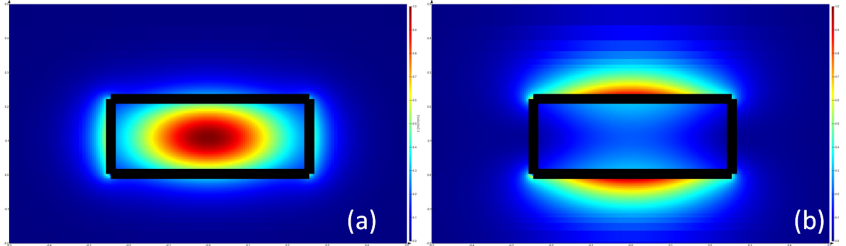

Fig. 3. Profile of the electric field for the (a) TE polarisation and (b) TM polarisation [18] in a 500-nm-wide and 220-nmhigh SOI waveguide.

was simulated as a function of the radius ( $\mathrm{r}$ ) and etching depth (h) of holes defined on the top of the waveguide as well as a function of the periodicity $(\Lambda)$ and length of the grating. In designing the optimal depth and radius of the holes great attention should be paid to the additional propagation losses introduced by the structure on both polarisations. Figure 4 shows the simulated propagation losses for the (a) TE and (b) TM mode. The theoretical losses were calculated with an Eigenmode Expansion (EME) solver from Lumerical Mode Solutions. To find the additional contribution to the losses introduced by the gratings only, the transmission spectra were normalised to a reference spectrum taken on a straight waveguide with identical length. Following the above simulations, the etching depth of the holes was fixed at $40 \mathrm{~nm}$ as this value makes the losses lower than $0.5 \mathrm{~dB} / \mathrm{cm}$ for both the TE and TM and yet provides sufficient interaction with the TM mode.

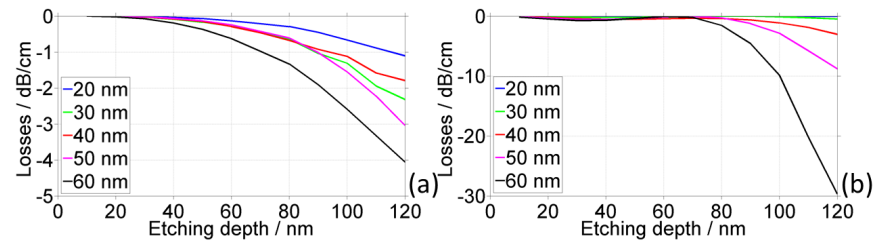

Fig. 4. Theoretical losses of the (a) TE and (b) TM polarised mode as a function of the grating hole radius and etching depth [18].

Figure 5 shows the simulated optical spectra for the TM polarised mode for a 40-nm deep circular top-grating as a function of the (a) grating length and (b) hole radius. As expected, and similarly to the sidewall gratings, an increase of the grating length or amplitude of the grating perturbation offer higher ERs.

The SOI waveguides investigated in this work consisted of a silicon core with height of $220 \mathrm{~nm}$ and width of $500 \mathrm{~nm}$. The TE gratings were defined as sinusoidal perturbation on the waveguide sidewall (insert of figure 2(b)) and the TM gratings as etched holes on the top of the waveguides (insert of figure 5(b)). The device pattern was defined using electron beam lithography on Hydrogen Silsequioxane (HSQ) and ZEP520A as the resist layers for the sidewall gratings and the top gratings, respectively. The patterns were transferred to the silicon core with the use of Reactive Ion Etching. In order to reduce the insertion loss, inverse tapers and polymer waveguides were used to couple the light in and out of the chip. The polymer waveguides were fabricated in SU8, with dimensions of $4.5 \mu \mathrm{m}$ in width and 3.5 $\mu m$ in height. The devices were coated with a $1 \mu \mathrm{m}$ thick plasma enhanced chemical vapour deposition (PECVD) $\mathrm{SiO}_{2}$ as a buffer layer. 

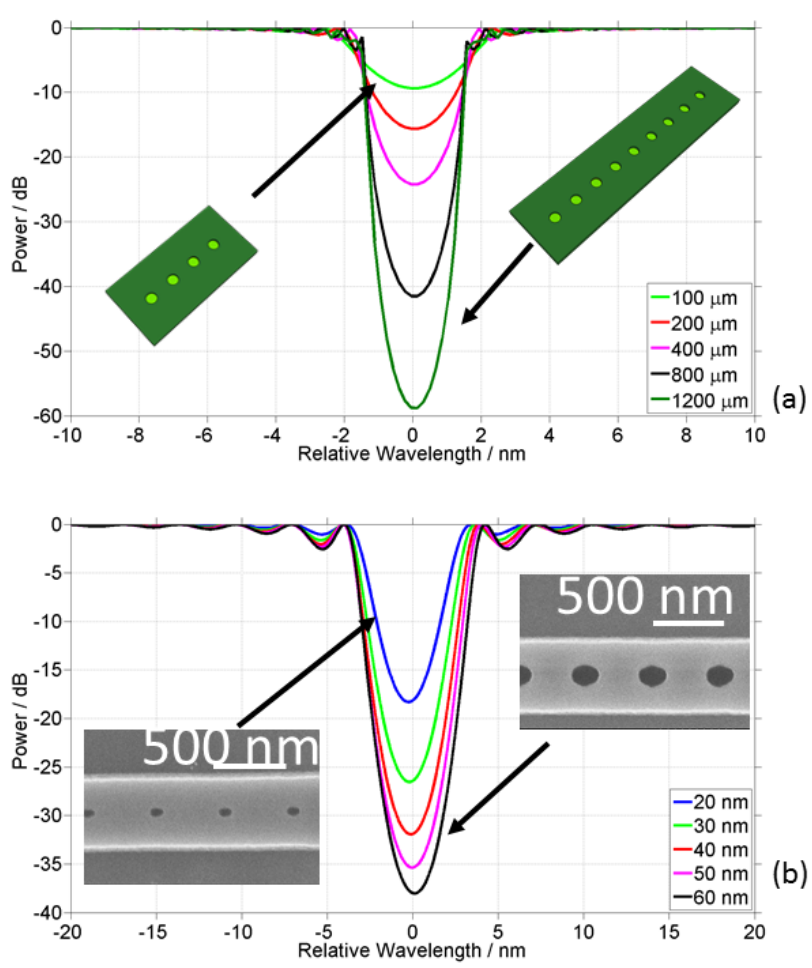

Fig. 5. The optical spectra of the TM polarisation on a single mode SOI waveguide with circular top gratings as a function of the (a) grating length with a hole radius of $40 \mathrm{~nm}$ and depth of $40 \mathrm{~nm}$ and (b) hole radius with a length of $670 \mu \mathrm{m}$ and depth of $40 \mathrm{~nm}$ [18].

The devices were measured using a standard end-fire rig transmission setup. A swept tunable laser (Agilent 8164b) was coupled through an in-line polarizer $(20 \mathrm{~dB})$ and a polarization maintaining (PM) lensed fibre into the input polymer waveguide. The output signal was coupled into a second PM lensed fibre and coupled directly to an optical spectrum analyser (OSA, Agilent 8614). To ensure accurate measurement of the grating stopband, the OSA was operated in high sensitivity mode $(-90 \mathrm{dBm}$ noise floor) with a resolution bandwidth of $0.1 \mathrm{~nm}$. An in-line polariser was used for the input fibre and no polarisation control was used for the output signal.

A number of gratings with different lengths were designed and fabricated to assess the performance of the top and sidewall gratings independently as well the combination of the two. Sidewall gratings with amplitude of $6 \mathrm{~nm}$ and top grating holes with etching depth of $40 \mathrm{~nm}$ and radius of $40 \mathrm{~nm}$ were chosen as TE and TM filters, respectively. The grating period was designed based on the Bragg relationship, $\Lambda=\lambda_{B} / 2 n_{\text {eff }}$, where $n_{\text {eff }}$ is the effective index of the optical mode. As for the design of the sidewall gratings, a sinusoidal perturbation was chosen to improve fabrication robustness [20]. These grating dimensions provide negligible propagation losses of less than $0.1 \mathrm{~dB} / \mathrm{cm}$ and grating kappa values of approximately $100 \mathrm{~cm}^{-1}$ for both polarisations, which translates into a theoretical ER approaching $60 \mathrm{~dB}$ on a $1 \mathrm{~mm}$-long grating. The periodicities for the two Bragg grating geometries were designed to be $318 \mathrm{~nm}$ and 448 $\mathrm{nm}$ for the TE and TM polarisation, respectively, due to the different effective index for the modes, so as to provide a stop-band centred around a wavelength of $1550 \mathrm{~nm}$.
Figure 6 shows the optical spectra for different grating lengths for the sidewall grating geometry when a TE polarised light is coupled to the device. The experimental data agree well with simulated ER values up to $30 \mathrm{~dB}$, after which the experimental ER value saturates because of polarisation scattering. The ER of the filter increases until it reaches a saturation value of approximately $30 \mathrm{~dB}$, which is well below the theoretical predictions. A similar trend was recorded with devices having a larger grating amplitude, which indicate that the $30 \mathrm{~dB}$ saturation value is independent of the grating $\mathrm{kL}$ product.

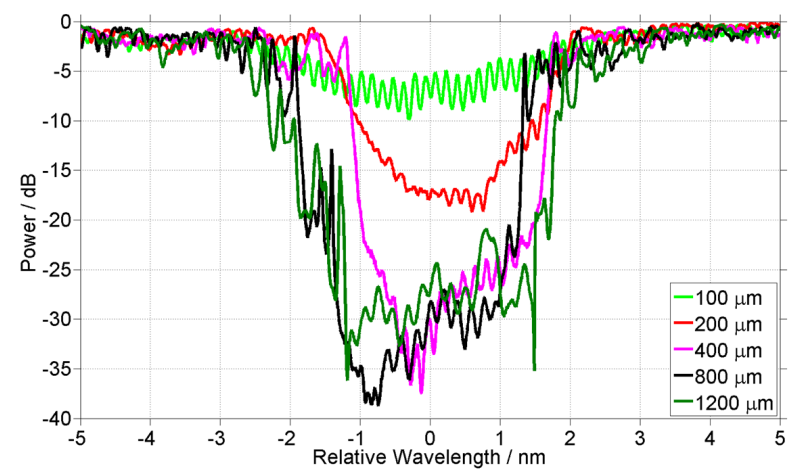

Fig. 6. Experimental transmission optical spectra of devices with $6 \mathrm{~nm}$-wide sidewall gratings, for a TE polarised input beam as a function of the grating length.

A similar set of measurements was performed on devices with top gratings for a TM polarised input beam. Similarly to the sidewall grating devices, the ER increases with the grating length until a saturation is reached around $25 \mathrm{~dB}$ as shown in figure 7 . Because the sidewall (top) grating is ineffective on the TM (TE) polarisation, the saturation value is a consequence of the nonperfect ER of the input polariser, which is approximately $30 \mathrm{~dB}$, and polarisation rotation effects occurring inside the device as a consequence of the strong scattering.

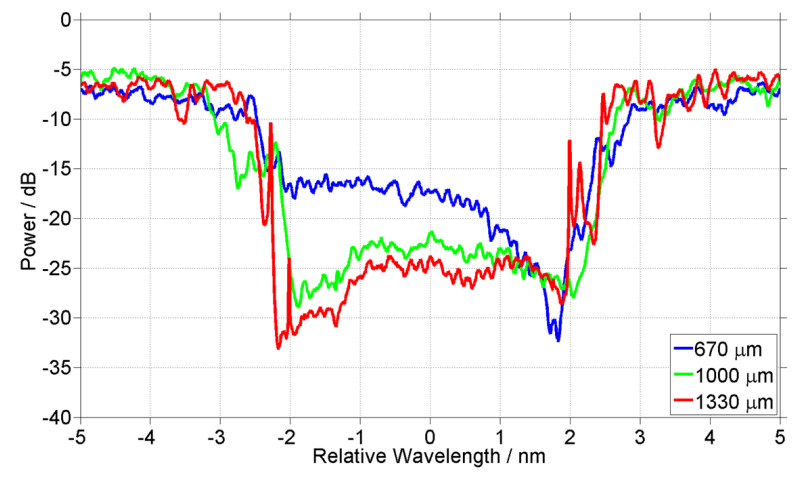

Fig. 7. Experimental transmission optical spectra of devices with circular top gratings with $40 \mathrm{~nm}$ depth and $40 \mathrm{~nm}$ radius for a TM polarised input beam as a function of the grating length.

The characterisation of the individual filters, was followed by the measurements of devices combining both filters on the same waveguide. Figures 8 shows the optical spectra for a TE polarised input signal for a $670 \mu \mathrm{m}$-long device combining both grating geometries. Because the top grating stop band is slightly 
narrower than the sidewall grating stop band, as the coupling coefficient for the TM filter was chosen to be smaller than the TE filter, the effect of combining the two geometries on the grating performance is clearly visible. In fact, where only the sidewall grating is effective (i.e. from approximately $1541 \mathrm{~nm}$ to 1546 $\mathrm{nm}$ ), the maximum ER is around $30 \mathrm{~dB}$, similarly to the values reported in Figure 6. Where the two stop bands overlay (i.e. from approximately $1537 \mathrm{~nm}$ to $1541 \mathrm{~nm}$ ), the ER substantially improves to a value of approximately $60 \mathrm{~dB}$, which is a value limited by the substrate scattering. When the two coupling coefficients are matched, the same high ER is observed for all the stop band as figure 9 shows. Similar figures in terms of maximum ER were measured when a TM polarised input beam was coupled to the device. It is worth noting that, although challenging, the $160 \mathrm{~nm}$ diameter of the top waveguide holes can be fabricated by DUV lithography [21].

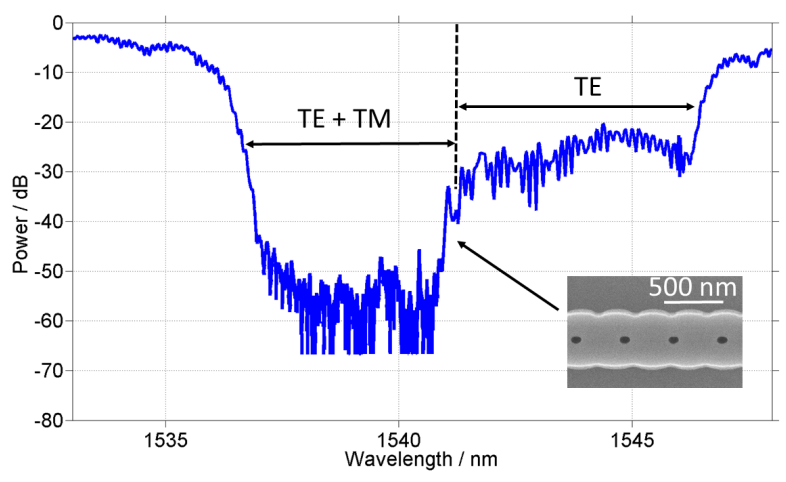

Fig. 8. The experimental transmission optical spectra of the combined sidewall and top grating with $40 \mathrm{~nm}$ radius for a TE polarised input signal. An increase of the ER is recorded in the spectral region where both TE and TM stopbands overlap (labelled 'TE+TM' in the figure).

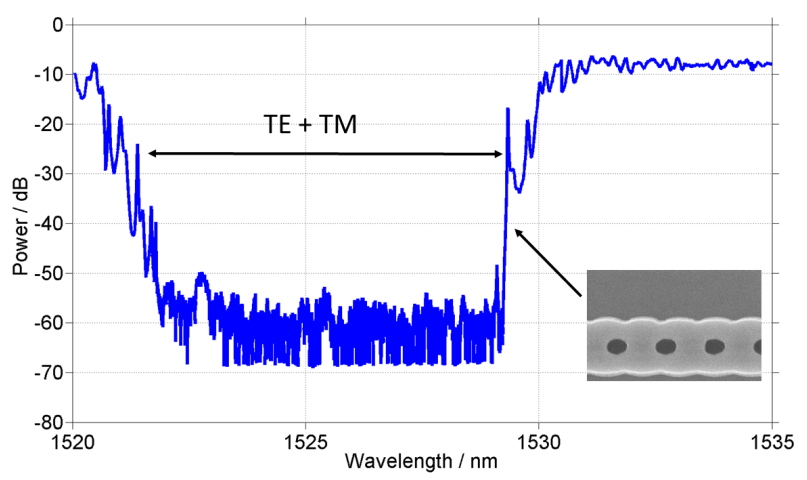

Fig. 9. The experimental transmission optical spectra of the combined sidewall and top grating with $80 \mathrm{~nm}$ radius for a TE polarised input signal. Because the width of the TM stopband is larger than that of the TE, the benefit produced by filtering the TM mode is visible over the whole stopband.

In this work, silicon waveguide sidewall and top Bragg grating filters were designed, fabricated and characterised as a function of their geometrical parameters. Because of the very different modal profile in single mode silicon waveguides, the sidewall geometry is mostly effective on the TE polarised mode while the top grating mostly interacts with the TM polarised mode. Combining these two geometries on the same waveguide allows the design of gratings that are effective on both polarisations thus substantially improving the grating ER. Experimental data show that the ER increases from $30 \mathrm{~dB}$, a limit given by the combination of polarisation scattering inside the waveguide and non-ideal input polarisers, to approximately $60 \mathrm{~dB}$. Such grating geometries also offer a solution for independent polarisation manipulation on-chip.

Funding:Engineering and Physical Sciences Research Council (EPSRC) (EP/L021129/1); H2020 Future and Emerging Technologies (FET) (323734); H2020 Excellent Science (645361).

Acknowledgment. The authors wish to thank the staff of the James Watt Nanofabrication Centre at the University of Glasgow. G. C. and C. K. acknowledge Fraunhofer Centre for Applied Photonics and EPSRC for the Ph.D. scholarship funding, respectively.

\section{REFERENCES}

1. D. T. H. Tan, K. Ikeda, R. E. Saperstein, B. Slutsky, and Y. Fainman, Opt. Lett. 33, 3013 (2008).

2. M. J. Collins, C. Xiong, I. H. Rey, T. D. Vo, J. He, S. Shahnia, C. Reardon, T. F. Krauss, M. J. Steel, a. S. Clark, and B. J. Eggleton, Nature communications 4, 2582 (2013).

3. S. Azzini, D. Grassani, M. J. Strain, M. Sorel, L. G. Helt, J. E. Sipe, M. Liscidini, M. Galli, and D. Bajoni, Opt. Express 20, 23100 (2012).

4. Y. de Koninck, F. Raineri, A. Bazin, R. Raj, G. Roelkens, and R. Baets, Opt. Lett. 38, 2496 (2013).

5. S. Keyvaninia, G. Roelkens, D. V. Thourhout, C. Jany, M. Lamponi, A. L. Liepvre, F. Lelarge, D. Make, G.-H. Duan, D. Bordel, and J.-M. Fedeli, Opt. Express 21, 3784 (2013).

6. C. Sima, J. C. Gates, H. L. Rogers, B. D. Snow, C. Holmes, M. N. Zervas, and P. G. R. Smith, Journal of Physics: Conference Series 276, 012089 (2011).

7. L. M. Rivas, M. J. Strain, D. Duchesne, A. Carballar, M. Sorel, R. Morandotti, and J. Azana, Opt. Lett. 33, 2425 (2008).

8. A. M. Prabhu, A. Tsay, Z. Han, and V. Van, IEEE Photonics Journal 2, 436 (2010).

9. F. Morichetti, A. Canciamilla, C. Ferrari, M. Torregiani, A. Melloni, and M. Martinelli, Phys. Rev. Lett. 104, 033902 (2010).

10. X. Wang, W. Shi, R. Vafaei, N. A. F. Jaeger, and L. Chrostowski, IEEE Photonics Technology Letters 23, 290 (2011).

11. M. J. Strain and M. Sorel, IEEE Journal of Quantum Electronics 46, 774 (2010).

12. M. Yamada and K. Sakuda, Applied optics 26, 3474 (1987).

13. J. R. Ong, R. Kumar, and S. Mookherjea, IEEE Photonics Technology Letters 25, 1543 (2013).

14. J. R. Ong, R. Kumar, and S. Mookherjea, Opt. Lett. 39, 4439 (2014).

15. N. C. Harris, D. Grassani, A. Simbula, M. Pant, M. Galli, T. Baehr-Jones, M. Hochberg, D. Englund, D. Bajoni, and C. Galland, Phys. Rev. X 4, 041047 (2014).

16. M. G. Saber, Z. Xing, D. Patel, E. El-Fiky, N. Abadia, Y. Wang, M. Jacques, M. Morsy-Osman, and D. Plant, IEEE Photonics Journal $\mathbf{9}$, 1 (2017).

17. M. Piekarek, D. Bonneau, S. Miki, T. Yamashita, M. Fujiwara, M. Sasaki, H. Terai, M. G. Tanner, C. M. Natarajan, R. H. Hadfield, J. L. O 'Brien, and M. G. Thompson, Opt. Lett. 42, 815 (2017).

18. Lumerical, "Lumerical Mode Solutions," https://www.lumerical.com/tcadproducts/mode/.

19. D. F. G. Gallagher and T. P. Felici, Proceedings of SPIE 4987, 69 (2003).

20. M. J. Strain, S. Thoms, D. S. Maclntyre, and M. Sorel, Opt. Lett. 39, 413 (2014).

21. R. Benevides, F. G. S. Santos, G. O. Luiz, G. S. Wiederhecker, and T. P. M. Alegre, Scientific Reports pp. 1-6 (2017). 